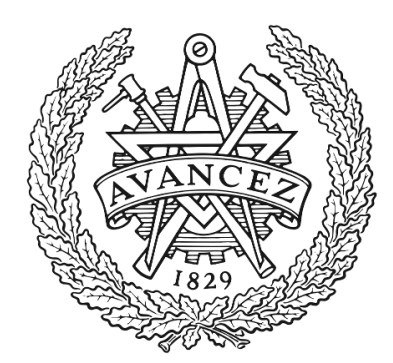

\title{
CHALMERS
}

UNIVERSITY OF TECHNOLOGY

\section{A Cosine Similarity Based Centralized Protection Scheme for DC Microgrids}

Downloaded from: https://research.chalmers.se, 2023-04-26 12:47 UTC

Citation for the original published paper (version of record):

Mohanty, R., Sahoo, S., Pradhan, A. et al (2021). A Cosine Similarity Based Centralized Protection Scheme for DC Microgrids. IEEE Journal of Emerging and Selected Topics in Power Electronics, 9(5): 5646-5656. http://dx.doi.org/10.1109/JESTPE.2021.3060587

N.B. When citing this work, cite the original published paper.

(O2021 IEEE. Personal use of this material is permitted.

However, permission to reprint/republish this material for advertising or promotional purposes 


\title{
A Cosine Similarity Based Centralized Protection Scheme for DC Microgrids
}

\author{
Rabindra Mohanty, Member, IEEE, Subham Sahoo, Member, IEEE, Ashok Kumar Pradhan, \\ Senior Member, IEEE and Frede Blaabjerg, Fellow, IEEE
}

\begin{abstract}
Unlike the phasor measurement based protection in AC systems, the protection of DC systems deals with complex fault transients which mandates the isolation of the faulted segment within few milliseconds as continued fault current leads to overheating issue in power electronic converters. To this end, several works have been suggested based on unit and nonunit protections for DC microgrids. Threshold selection and protection coordination are the challenges associated with nonunit protection. Similarly, communication delay and link failure limit the application of unit protection. To address these issues, this paper presents a robust centralized protection scheme for DC microgrids, which is resilient to communication delay and link failure. It uses current of each line segment to compute the similarity of current change at both ends of the line segment to derive the protection decision. To overcome the communication failure from one end of the line segment or even from multiple segments, the proposed method uses data from adjacent segments to derive the protection decision correctly. Using PSCAD/EMTDC environment, the performance of the proposed method is evaluated for various cases and compared with available techniques. Finally, the accuracy of the protection algorithm is validated under experimental conditions.
\end{abstract}

Index Terms-Centralized protection, communication, cosine similarity, DC microgrid, fault analysis.

\section{INTRODUCTION}

W ITH advancement in power electronic converters and communication infrastructure, microgrid technologies promote the integration of distributed generation(DG) with energy storage sources (ESSs) to provide efficient and quality power to customers [1]. Today, DC microgrids have emerged as a promising architecture with majority of electronic loads consuming DC power and operability of DC producing renewable sources like photovoltaic (PV) array, fuel cell and ESSs. DC mains and feeders reduce the number of conversion stages, as compared to $\mathrm{AC}$ and improve the efficiency [2]. Without synchronization and reactive power control requirements, operation of multiple parallel power electronic converter inter-

This work was supported in part by Energy Area of Advance at Chalmers University of Technology, Sweden and in part by THE VELUX FOUNDATIONS under the VILLUM Investigator Grant - REPEPS (Award Ref. No.: 00016591).

R. Mohanty is with the Division of Electric Power Engineering, Chalmers University of Technology, Gothenburg, Sweden 41296 (e-mail: rabindra@chalmers.se)

S. Sahoo and F. Blaabjerg are with the Department of Energy Technology, Aalborg University, Aalborg East, 9220, Denmark (e-mail: sssa@et.aau.dk and fbl@et.aau.dk)

A. K. Pradhan is with the Department of Electrical Engineering, Indian Institute of Technology Kharagpur, India 712302 (e-mail: akpradhan@ee.iitkgp.ac.in) faced renewable energy source (RES) becomes an easy task in DC microgrids as compared to the AC counterparts [3].

One of the primary challenges in DC microgrids is the lack of an effective protection solution [4]. With the occurrence of a fault, the DC-link capacitor of the power electronic converter discharges rapidly causing the DC bus voltage to drop sharply. Further, the energy stored in the cable inductance also discharges through the freewheeling diodes of the converters resulting in a high magnitude fault current within a very short interval of time [5]. The converters operate as uncontrolled rectifiers (forward biasing of anti-parallel diodes) during fault conditions with the fault current still being fed from the input side of the converter [6]. This may cause an unstable operation of the system and even damage the power semiconductor switches in the converters unless the fault is cleared.

The existing protection schemes in DC microgrid can be categorized into non-unit and unit protections. Local measurement based non-unit protection schemes are presented for DC microgrids in [7]-[9], which do not require communication. However, its performance is influenced by factors such as requirement of high bandwidth measurement devices [7], selection of thresholds [8] and can be accommodated only for long distance DC line based systems [9]. Additionally, these schemes do not guarantee selectivity. On the other hand, unit protection schemes exploit data from both ends of the line to be protected by overcoming the abovementioned issues. Regardless of its advantages, high resistance fault could be an issue for current differential [10], [11] and current directional [12] based unit protection schemes. The performance of the differential scheme is highly dependent on the current threshold, since the operating current may not exceed it during high resistance fault. Similarly, the direction of fault current during high resistance fault does not alter, as the pre-fault current can be more than the faulted current under heavy loading condition. In addition, communication delay and communication link failure limit the application of unit protection schemes.

Intelligent electronic devices (IEDs) are used for protection and automation with the support of IEC 61850 protocol [13]. Since IEC 61850 is known for its flexibility due to its capability of decoupling domain-specific models from the communication stack, it has been adopted as an international communication medium to monitor, control and measure the physical processes in a microgrid. A centralized protection scheme compatible with IEC 61850 protocol has the capability to provide promising solution for microgrids, that can deal with the above stated issues associated with both non-unit and 


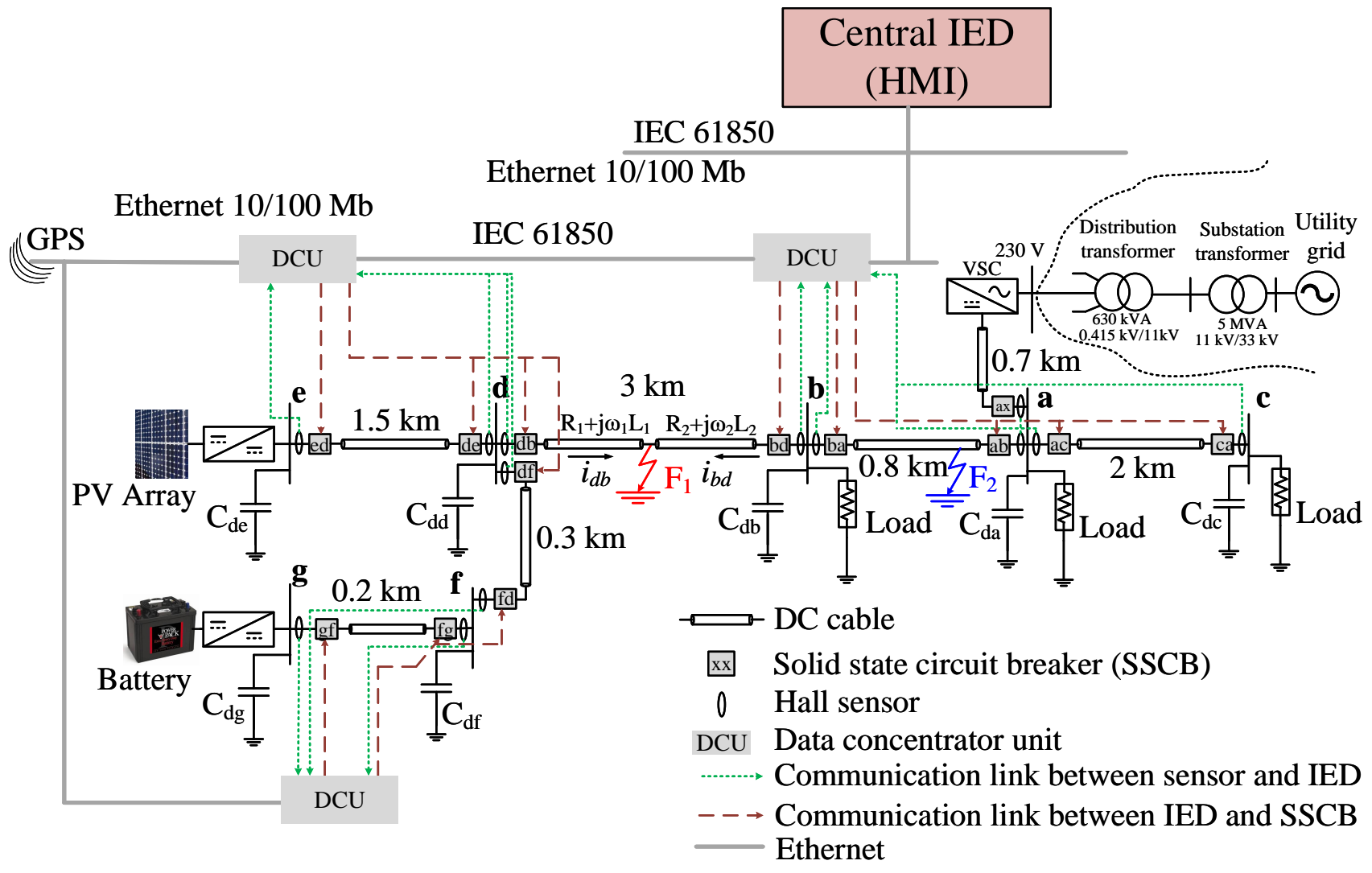

Fig. 1. Single line diagram of the considered grid-tied DC microgrid.

unit protection schemes. In [5], [14], [15], similar protection schemes have been proposed for DC microgrids. A current derivative based protection algorithm is proposed in [5]. However, the selection of threshold in a multi-converter system can become a challenging task as low impedance of DC line segment can offer high rate of change of current even during non-faulted conditions. Further, a differential current based protection approach is used in [14], [15], where the accuracy of these schemes can not be guaranteed for high resistance faults. Moreover, the effects of communication delay and link failure on the performance of these methodologies are not studied.

Accuracy in the operation of any protection algorithm depends on proper fault characterization of the system. An accurate mathematical model of fault in a DC system is difficult to formulate as it is nonlinear, time varying and strongly coupled with control strategy of converters [16]. Hence, this mandates the design of a high-resolution robust protection scheme using statistical comparison of the magnitude and direction of current information from both ends accounting cyber adversities, such as communication delay and link failure.

To address these issues, a centralized protection scheme based on the similarity of current change over a window of both ends of a line segment is proposed for DC microgrids. The cosine similarity index $(C S I)$ is used to differentiate the internal and external faults. Unlike sample-to-sample based approach [16], a window of data set is considered to overcome the effect of communication delay in the protection scheme.
The current from each line segment is communicated to a central IED using IEC 61850 protocol for DC microgrid. The central IED overcomes the issue associated with communication link failure and delay from any location using data of adjacent line segments and generates a trip signal accurately for the corresponding breaker in the faulted segment. Robustness in the performance of the proposed method is evaluated for various cases including high resistance fault, communication delay and link failure in PSCAD/EMTDC environment. Finally, the performance of the proposed algorithm is validated under experimental conditions.

The rest of the paper is organized as follows. In section II, brief descriptions of DC microgrid, different stages during fault and communication infrastructure are provided. A comparative study of the existing protection schemes is illustrated in section III. The cosine similarity based proposed method is presented in section IV. The performance of the proposed method is tested for different cases using data from simulation and experimental setup in section V and VI respectively. Section VII and VIII present the discussion and conclusion of the work respectively.

\section{Fault Analysis in DC Microgrid}

A $30 \mathrm{~kW}, 350 \mathrm{~V}$ DC microgrid (as shown in Fig. 1) is considered, which consists of DC-DC boost converters at PV array terminal, a battery with bidirectional DC-DC buckboost converter and a front-end voltage source converter (VSC) connected to the utility grid. The VSC is connected to single 
TABLE I

FAULT CURRENT RESPONSE OF CONVERTERS IN DC MiCROGRID

\begin{tabular}{|l|l|l|l|l|}
\hline \multirow{2}{*}{$\begin{array}{c}\text { DC fault } \\
\text { current response }\end{array}$} & \multicolumn{4}{|c|}{ PG fault } \\
\cline { 2 - 5 } & VSC & $\begin{array}{c}\text { Boost } \\
\text { converter }\end{array}$ & $\begin{array}{c}\text { Buck } \\
\text { converter }\end{array}$ & $\begin{array}{c}\text { Buck-boost } \\
\text { converter }\end{array}$ \\
\hline Stage 1 & Yes & Yes & Yes & Yes \\
\hline Stage 2 & Yes & Yes & Yes & Yes \\
\hline Stage 3 & Yes & Yes & No & No \\
\hline
\end{tabular}

phase of low voltage side of distribution transformer of 630 $\mathrm{kVA}, 50 \mathrm{~Hz}, 0.415 / 11 \mathrm{kV}$. Finally, it is connected to the main grid via a substation transformer of $5 \mathrm{MVA}, 50 \mathrm{~Hz}, 11 / 33$ $\mathrm{kV}$. Loads in the microgrid are primarily supplied by local PV arrays and under heavy loading conditions, the remaining power is extracted from the grid. On the other hand, converter interfaced with battery operates in voltage-controlled mode to regulate generation-demand imbalance during islanded mode of operation. The capacitance shown at each bus is the equivalent of DC link capacitor and cable as $\mathrm{C}_{d x}$, where the subscript $x$ represents the bus number. The front-end VSC maintains the voltage at the point of common coupling (PCC) for grid connected mode of operation. The PV arrays inject maximum power into the grid with the help of a maximum power point tracking (MPPT) control strategy. Two cascaded PI control loops create a modulation signal in a complementary fashion to control the switches in bidirectional DC-DC converter which operates in voltage controlled mode.

During a fault on the DC side, the transient response affects three counterparts; such as Stage 1: capacitor discharge or natural response, Stage 2: freewheeling diode operation, and Stage 3: current fed from the grid. A detailed response in time domain of the nonlinear circuit is analyzed for different time periods in [6], [17]. The occurrence of these stages depend on the converter topology and the type of fault [6]. Table I provides the stages of transient response for VSC and DCDC converters during pole-to-ground (PG) faults in a unipolar DC microgrid. It is observed that Stage 1 and Stage 2 are present in all cases during fault. The protection system is expected to operate within the rise time of current (during Stage 1) to protect the switches in the converters from overheating stress in Stage 2 and Stage 3.

Several communication protocols are developed to exchange the information between IEDs and power equipment in a microgrid. In electrical substation, distributed network protocol(DNP3), Modbus TCP/IP and protocols compliant with IEC 61850 are widely used. The IEC-61850 standard is part of the IEC technical committee 57 (IEC-TC-57) that deals with information model and logical nodes (LNs) including DGs, electrical connection points, controllers, generators, power converters, measurement and protection devices [18]. IEC 61850 features include data modeling, reporting, fast transfer (GOOSE and GSSE), setting groups, sampling data value, command configuration and data storage. The data object and attributes of IEC 61850 monitor the measurement, breaker status and setting group status in the considered DC microgrid in Fig. 1.

\section{Comparative Evaluation of Existing PROTECTION SCHEMES}

The current derivative $\left(\frac{d i}{d t}\right)$ based protection is simple and computationally effective solution for DC microgrids [5], [8], [19]. Such methods take protection decision within few milliseconds. However, the high-frequency switching ripples complicate the trip decision of current derivative based protection methods. Fig. 3 shows different ripple frequencies and magnitudes of current for various switching frequencies $N_{s}$ at the output of a DC-DC buck converter. For $4 \mathrm{kHz}$ sampling, the magnitudes of current gradient are $18 \mathrm{~A} / 250 \mu \mathrm{s}$ and 32 $\mathrm{A} / 250 \mu \mathrm{s}$ for $N_{s}=5 \mathrm{kHz}$ and $N_{s}=1.5 \mathrm{kHz}$ respectively. These values are significantly high, which can easily exceed the thresholds.

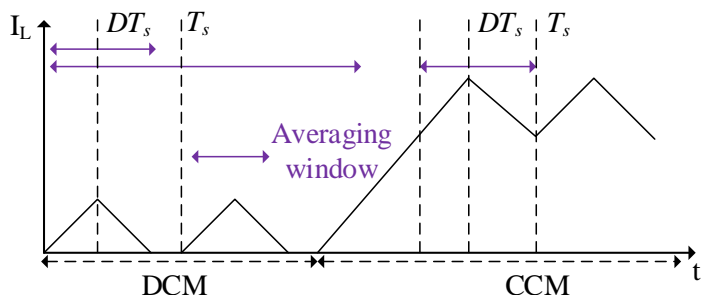

Fig. 2. CCM/DCM operation modes for a DC-DC boost converter.

Furthermore as shown in Fig. 2, DCM/CCM mode are primarily dependent on the loading condition in the output, where the converter either operates at DCM or CCM with respect to the critical $R_{\text {crit }}$ value. Usually, the existing protection mechanisms to detect a fault are based on averaging mechanism for signals which does not contain any switching ripples from the sources, since they have been formulated for standard DC supplies. However, when the said fault detection philosophies are tested for power electronic converters with switching ripples in the current, the following issues arise:

- the rate of change in current is not solely dependent on the fault but also dependent on other factors such as switching frequency, variable loading condition, voltage levels, etc.

- the design of averaging window with a fixed threshold to generate a decision under faults may be tricky (as shown in Fig. 2), since the average values could vary for DCM/CCM modes (under different loading conditions), different window sizes, etc. As a result, it could often lead to false tripping of converters for a fixed threshold averaging mechanism to detect faults.

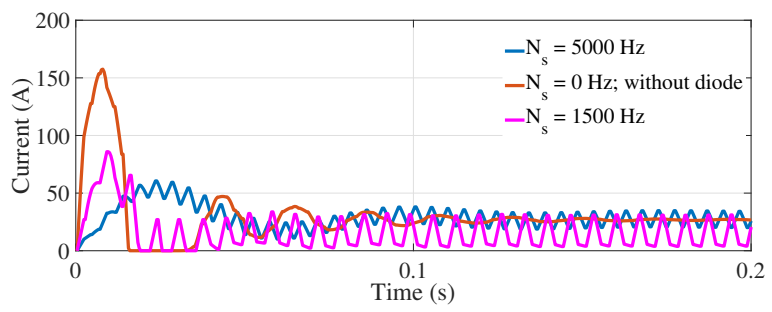

Fig. 3. Current response of DC-DC buck converter with different switching frequencies $N_{s}$. 
TABLE II

Comparative Assessment of EXIsting Protection Techniques

\begin{tabular}{|c|c|c|}
\hline Protection methods & Work done & Remarks \\
\hline Methods based on $\frac{d i}{d t}[5],[8],[19]$ & $\begin{array}{l}\text { - Low resistance fault is detected by current } \\
\text { derivative. } \\
\text { - High resistance fault detected by second order } \\
\text { derivative and current differential. }\end{array}$ & $\begin{array}{l}\text { - Requires both voltage and current measurements. } \\
\text { - Each line segment needs two IEDs at both ends } \\
\text { to derive the protection decision. } \\
\text { - Vulnerable to non-fault disturbance that causes } \\
\text { higher current derivatives. } \\
\text { - IED coordination is not discussed in local data } \\
\text { based protections. }\end{array}$ \\
\hline $\begin{array}{l}\text { Superimposed current } \\
\text { based unit protection [20] } \\
\text { Method based on } \\
\text { similarity index [16] }\end{array}$ & $\begin{array}{l}\text { - Superimposed current at both ends of the line } \\
\text { segment. } \\
\text { - Cosine similarity of the transient waveform } \\
\text { distinguishes the internal and external faults. }\end{array}$ & $\begin{array}{l}\text { - Method needs two IEDs [20] and one IED [16] } \\
\text { in each line segment to be protected. } \\
\text { - The superimposed component and similarity index } \\
\text { cannot be derived for a communication link failure. }\end{array}$ \\
\hline Centralized protection [14], [15] & $\begin{array}{l}\text { - Current differential and moving average } \\
\text { based protection. }\end{array}$ & $\begin{array}{l}\text { - The current differential does not work } \\
\text { for communication link failure. } \\
\text { - Communication delay causes the maloperation } \\
\text { of differential technique for external faults. }\end{array}$ \\
\hline Proposed protection scheme & $\begin{array}{l}\text { - Cosine similarity index of two independent } \\
\text { data windows of average } \frac{d i}{d t} \text {. } \\
\text { - Validated in experimental setup. }\end{array}$ & $\begin{array}{l}\text { - Current measurement only. } \\
\text { - One central IED is required instead } \\
\text { of IED at each line segments. } \\
\text { - Robustness towards communication delay } \\
\text { and link failure. } \\
\text { - The protection decision is unaffected } \\
\text { in presence of ripples due to high-frequency } \\
\text { switching and for high resistance fault. }\end{array}$ \\
\hline
\end{tabular}

In the microgrid with short line lengths, the $\frac{d i}{d t}$ of adjacent segments may exceed the predefined threshold values. Although, the peak depends on the corresponding bus voltage and inductance of the faulted path, the local data based protection using current derivative operates at the moment the derivative exceeds the predefined threshold irrespective of the peak value. For example, a fault in the middle of the line segment-fd (refer Fig. 1) causes the $\frac{d i}{d t}$ at SSCB-gf and SSCB-fd above the threshold $(25 \mathrm{~A} / 250 \mu \mathrm{s})$ as shown in Fig. 4. Thus, local data based $\frac{d i}{d t}$ fails to identify the correct faulted section resulting into a selectivity issue. The above mentioned problem is more prominent in case of small capacitor (or no capacitor), which is present between the buses.

The selectivity issue in local data based technique is solved using communication assisted current differential, current directional and centralized protections in DC microgrid. High resistance fault is a problem for current differential and directional protection schemes. For example, the operating current $\left(\mathrm{I}_{\text {diff }}\right)$ in the current differential technique goes below the threshold for fault resistance more than $5.5 \Omega$, as evident from Fig. 5. Similarly, the direction of current has not changed during the fault with fault resistance more than $5 \Omega$, as shown in Fig. 6. As a result, such faults cannot be identified correctly.

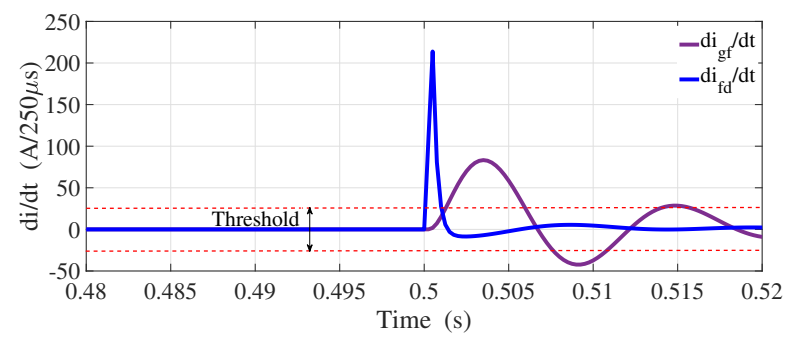

Fig. 4. Current derivatives at SSCB-gf and SSCB-fd for the fault in line segment-fd.

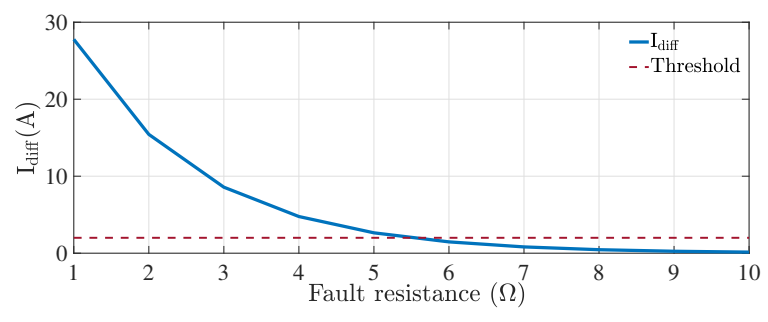

Fig. 5. Current difference during high resistance internal fault.

Note that the rated current for a $30 \mathrm{~kW}, 350 \mathrm{~V}$ DC system is 85.7 A. The fault resistance that causes the current less than the rated value is considered as high resistance fault. In this case, the current of 85.7 A corresponds to $4 \Omega$. Thus, the fault resistance of $5 \Omega$ or more is considered as high resistance fault.

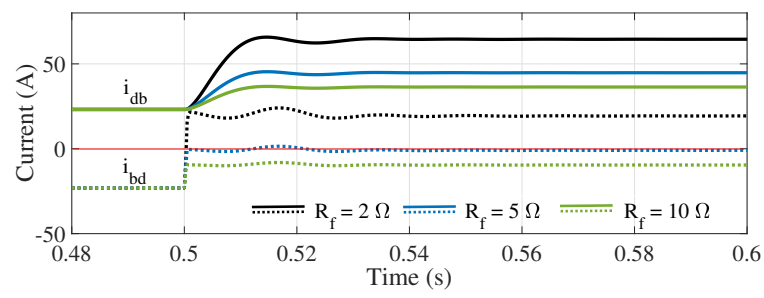

Fig. 6. Currents of both ends during high resistance fault $F_{1}$.

Communication delay $\left(\epsilon_{n}\right)$ results in maloperation of communication based protections schemes such as differential scheme during an external fault. To illustrate this issue more clearly, a pole-to-ground fault is created in line segment-ab in Fig. 1 (considered as an external fault to the segment-bd), the current $i_{d b}$ and $i_{b d}$ are observed, and their sum is plotted in Fig. 7. The $i_{b d}$ is communicated with a time delay, $\epsilon_{t}=1$ ms with respect to $i_{d b}$. In such a case, the current difference 
enters into operating region for a certain period of time as shown in Fig. 7. The threshold of the current differential in a DC microgrid is considered as $\eta I_{n}$ [21], where $\eta$ is the reliability coefficient whose value is in between $6 \%$ to $20 \%$ and $I_{n}$ is the nominal current of the line segment, i.e. $85.7 \mathrm{~A}$ in this case. Under these circumstances, the proposed methods in [14], [15], [21] will maloperate.

id

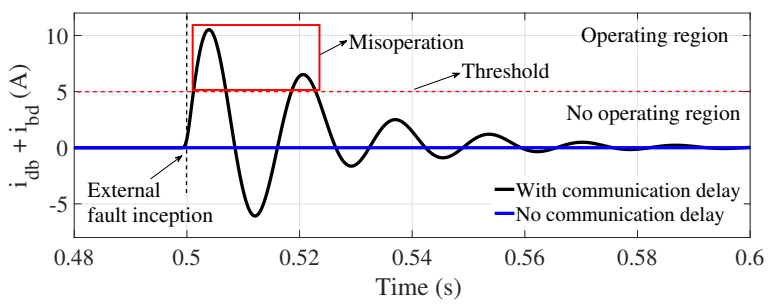

Fig. 7. Current differential with and without communication delay during a fault in adjacent line $a-b$.

The sign of $\frac{d i}{d t}$ has the inherent capability of distinguishing faults in forward and reverse directions. The $\frac{d i}{d t}$ is positive for forward fault and negative for reverse fault, provided the prefault power flow is in forward direction. However, the sign of $\frac{d i}{d t}$ needs to be communicated to the remote end (and viceversa) to identify the internal fault which requires communication link, similar to unit protection. In summary, the local $\frac{d i}{d t}$ based protections without communication have issues with threshold setting and IEDs coordination. The communication based unit protections are associated with issues like communication failure and delay. Similarity index based protection method in [16] is not evaluated for communication link failure. In addition, it needs at least one IED for each line segment in the DC microgrids which increases the cost. All the issues with the existing protection strategies are provided in Table II. Furthermore, the advantages behind employing the proposed strategy is also detailed in the same table.

\section{Proposed Protection Scheme}

The DC microgrid considered in this work is shown in Fig. 1. The microgrid is divided into different protection segments and each segment consists of a solid-state circuit breaker (SSCB) at each end. A central IED is installed for the entire microgrid. The cyber layer, shown in Fig. 1, is realized using SimEvents based realistic communication model to simulate all the intermittent parameters associated with the communication network [22]. Currents of both ends of a line segment are communicated to the IED (highlighted in Fig. 1) and the rate of change of current at each end is obtained.

\section{A. Fault detection}

The collected line currents at IED are sampled at a rate of $4 \mathrm{kHz}$. A disturbance index $(h)$ is calculated using current information from the following relation

$$
h=\frac{1}{N \Delta t}\left(\sum_{n=1}^{N}\left|i_{n+1}-i_{n}\right|\right)
$$

where $i_{n}$ corresponds to sample value of current for $\mathrm{n}^{\text {th }}$ instant with $\mathrm{N}=4$ as four samples in $1 \mathrm{~ms}, \Delta t$ is the sampling interval. The absolute value of the current difference is considered in (1) to monitor the magnitude change only. When $h>\zeta$ (threshold), a fault is ensured; else, it suggests a normal condition. The threshold $\zeta$ is calculated as:

$\zeta=\frac{1}{N \Delta t}\left(\sum_{n=1}^{N}\left|i_{n+1}-i_{n}\right|\right)=\frac{1 \times 5 A}{4 \times 250 \times 10^{-6} s}=5000 \mathrm{~A} / \mathrm{s}$

Considering high security of IED operation $\sum_{n=1}^{N}\left|i_{n+1}-i_{n}\right|$ $=5 \mathrm{~A}$ is set, where $\sum_{n=1}^{N}\left|i_{n+1}-i_{n}\right|$ represents the sum of four consecutive sample-to-sample differences. It is to be noted that the disturbance index becomes high even for load change or any other switching phenomena. Fig. 8 shows the current response and $\mathrm{N} h \Delta t$ for a sudden change in load (which may confuse relay with fault) as $h$ exceeds $\zeta$. This is similar to a fault detector in available numerical relays which triggers the next step on the decision process.

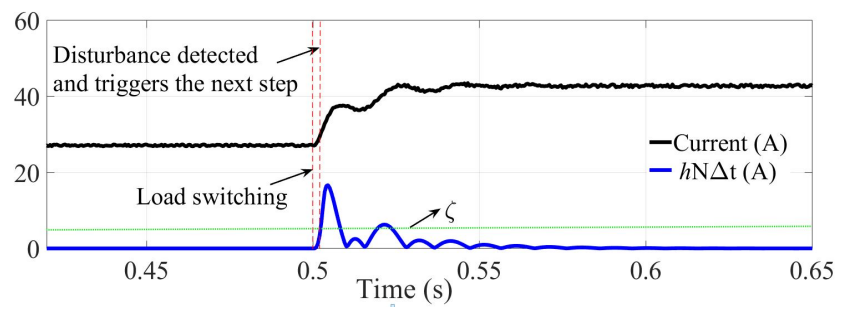

Fig. 8. Change in current and $h \mathrm{~N} \Delta \mathrm{t}$ with increase in load.

\section{B. Current response during an internal fault}

In Stage 1 of fault analysis, the RLC response of the DC line current $i_{d b}$ at bus-d in frequency domain during $F_{1}$ can be written as:

$$
i_{d b}(s)=\frac{\frac{V_{d d}(0)}{L_{1}}+i_{d b}(0)}{s^{2}+\frac{R_{1}}{L_{1}} s+\frac{1}{L_{1} C_{d}}}
$$

where $V_{d d}(0)$ is the voltage across the $C_{d d}$ at bus-d and $i_{d b}(0)$ is the current through $L_{1}$ at the instant of fault inception. Fault component of current in time domain is given by:

$$
\begin{array}{r}
i_{d b}^{F_{1}}(t)=\frac{V_{d d}(0)}{L_{1}\left(m_{2}-m_{1}\right)}\left(e^{-m_{1} t}-e^{-m_{2} t}\right) \\
+\frac{i_{d b}(0)}{m_{2}-m_{1}}\left(-m_{1} e^{-m_{1} t}+m_{2} e^{-m_{2} t}\right)
\end{array}
$$

where $m_{1}=-\alpha_{1}+\sqrt{\alpha_{1}^{2}-\omega_{0}^{2}}, m_{2}=-\alpha_{1}-\sqrt{\alpha_{1}^{2}-\omega_{0}^{2}}$ and, $\alpha_{1}$ $=R_{1} / 2 L_{1}, \omega_{1}=\sqrt{\frac{1}{L_{1} C_{d d}}-\left(\frac{R_{1}}{2 L_{1}}\right)^{2}}$ and $\omega_{0}=\sqrt{\alpha_{1}^{2}+\omega_{1}^{2}}$. The time derivative of (4) at $\mathrm{t}=0^{+}$at the instant of fault inception will be:

$$
\frac{d i_{d b}(t)}{d t}=\frac{V_{d d}(0)}{L_{1}}-2 \alpha_{1} i_{d b}(0)
$$

Using (5), it can be concluded that the rate of change of current depends on the initial conditions of voltage across the DC link capacitor and line current just before the fault. 
In a similar way at bus-b, for the fault $F_{1}$, the rate of change of line current $i_{b d}$ will be:

$$
\frac{d i_{b d}(t)}{d t}=\frac{V_{d b}(0)}{L_{2}}+\frac{R_{2}}{L_{2}} i_{b d}(0)
$$

Remark I: As a convention with the direction of currents as shown in Fig. 1, it is clear that for internal fault $F_{1}$, the $\frac{d i_{d b}(t)}{d t}$ and $\frac{d i_{b d}(t)}{d t}$ are positive.

\section{Current response during an external fault}

Remark II: In case of an external fault $F_{2}$ as shown in Fig. 1, the $\frac{d i_{d b}(t)}{d t}$ and $\frac{d i_{b d}(t)}{d t}$ are equal in magnitude as well as their rate of changes. However, the sign will be opposite, e.g. $\frac{d i_{d b}(t)}{d t}=-\frac{d i_{b d}(t)}{d t}$. Thus,

$$
\begin{aligned}
\frac{d i_{b d}(t)}{d t}= & \frac{d\left(-i_{d b}(t)\right)}{d t}=\frac{-d i_{d b}(t)}{d t} \\
& =-\frac{V_{d d}(0)}{L_{1}}+\frac{R_{1}}{L_{1}} i_{d b}(0)
\end{aligned}
$$

\section{Cosine similarity index (CSI) based protection decision}

In the above subsections, the direction of current during transient is analyzed. A simple threshold based protection decision is challenging for power-electronic converters due to distortion and high-frequency ripples during transients. Therefore, a window of 2 ms (i.e. 8 samples $\times \frac{1}{4000} \mathrm{~s}$ ) is considered as shown in Fig. 9. The average of the rate of change of current in a window is obtained at each end of a line segment. The alikeness of two data windows of both ends of the line segment is measured using cosine similarity. The idea of cosine similarity used in the proposed method is analogous to the $C S I$ of two non-zero vectors in a dot product, which has been widely used in data mining applications [23], [24].

Using two consecutive samples, the rate of change of current is obtained in a window of $2 \mathrm{~ms} ; 7$ number of $\frac{d i}{d t}$ samples in a window (in Fig. 9). where $d i_{d b}(t) / d t \stackrel{d t}{=}\left(i_{d b}\left(t_{2}\right)-i_{d b}\left(t_{1}\right)\right) /\left(t_{2}-t_{1}\right)$. For a window of data set, $\mathrm{X}=$ average $\left\{x_{1}, x_{2}, x_{3}, \ldots x_{7}\right\}=$ average $\left\{\frac{d i_{d b 1}}{d t}, \frac{d i_{d b 2}}{d t}, \frac{d i_{d b 3}}{d t}, \ldots \frac{d i_{d b 7}}{d t}\right\}$ and $\mathrm{Y}=$ average $\left\{y_{1}, y_{2}, y_{3}, \ldots y_{7}\right\}=$ average $\left\{\frac{d i_{b d 1}}{d t}, \frac{d i_{b d 2}}{d t}, \frac{d i_{b d 3}}{d t}, \ldots \frac{d i_{b d 7}}{d t}\right\}$, $C S I$ can be calculated as below:

$C S I=\frac{X . Y}{|X||Y|}=\frac{\frac{1}{7} \sum_{n=1}^{7} x_{n} \cdot \frac{1}{7} \sum_{n=1}^{7} y_{n}}{\left|\frac{1}{7} \sum_{n=1}^{7} x_{n}\right|\left|\frac{1}{7} \sum_{n=1}^{7} y_{n}\right|}=\frac{\sum_{n=1}^{7} x_{n} \cdot \sum_{n=1}^{7} y_{n}}{\left|\sum_{n=1}^{7} x_{n}\right|\left|\sum_{n=1}^{7} y_{n}\right|}$

For an external fault $F_{2}, X \approx \sum_{n=1}^{7} x_{n}$ and $Y \approx \sum_{n=1}^{7} y_{n}$ in the line segment-db are positive and negative respectively, using Remark II. However, for internal fault $F_{1}$, both $X$ and $Y$ are positive using Remark I. The similarity index output can be summarized by:

$$
C S I= \begin{cases}+1, & \text { if }, \text { internal fault } \\ -1, & \text { if }, \text { external }\end{cases}
$$

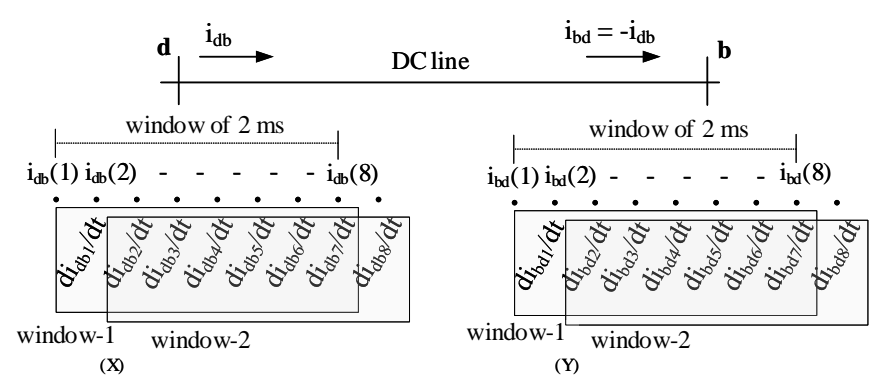

Fig. 9. Independent data windows of both ends of the line segment-bd.

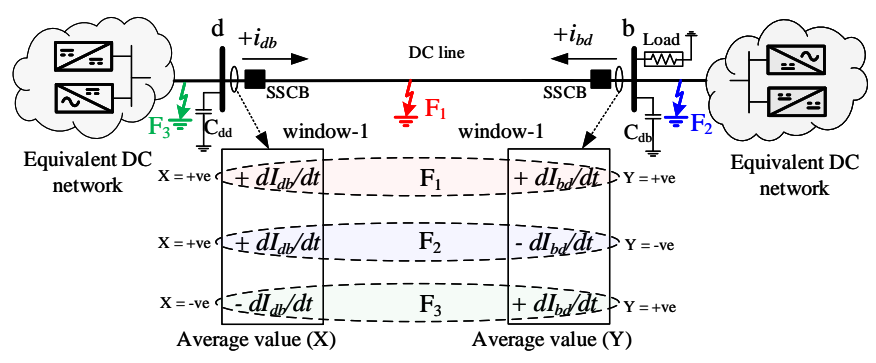

Fig. 10. Similarity of windows during internal and external faults.

The similarity of change in current at both ends of the line segment, $\mathrm{X}$ and $\mathrm{Y}$ in (8) is shown in Fig. 10. For the internal fault $\left(F_{1}\right), C S I$ comes out to be +1 as both $\mathrm{X}$ and $\mathrm{Y}$ are positive. However, $C S I$ is -1 for the external faults $\left(F_{2}\right.$ and $\left.F_{3}\right)$ as $\mathrm{X}$ and $\mathrm{Y}$ are opposite in sign. A detailed flowchart of the proposed protection scheme is provided in Fig. 11.

E. Communication link failure from one end of the faulted segment

The fault $F_{1}$ can not be confirmed from $\mathrm{X}$ and $\mathrm{Y}$ of line segment-db if $i_{b d}$ is not communicated due to communication link failure as shown in Fig. 12. In this case, the IED uses the similarity of $i_{d b}$ and $i_{a b}$ to generate a trip decision. The trip signal will be generated if $\left|i_{d b}\right|-\left|i_{\text {ba }}\right|>\left|i_{\text {load_b } b}\right|$, where $i_{\text {load_b }}$ is the rated load current at bus-b. Thus, the proposed method performs satisfactorily, even if communication link fails from one end of a line segment.

\section{F. Performance during multiple link failure}

In case of multiple communication link failure, a fault can be identified using the available information of rate of change of current. For example, fault $F_{1}$ can be identified using the $C S I$ of $i_{d b}$ and $i_{a b}$ in case of communication failure of $i_{b d}$ and $i_{b a}$ (refer Fig. 12). Under such circumstances, the adjacent segment including the faulted one will be isolated. The trip decision will be derived based on $C S I=+1$ and for the fault $F_{1}$, the line segment-db and ab will be isolated.

It is worth notifying that the proposed method identifies the exact faulted segment when one communication channel 


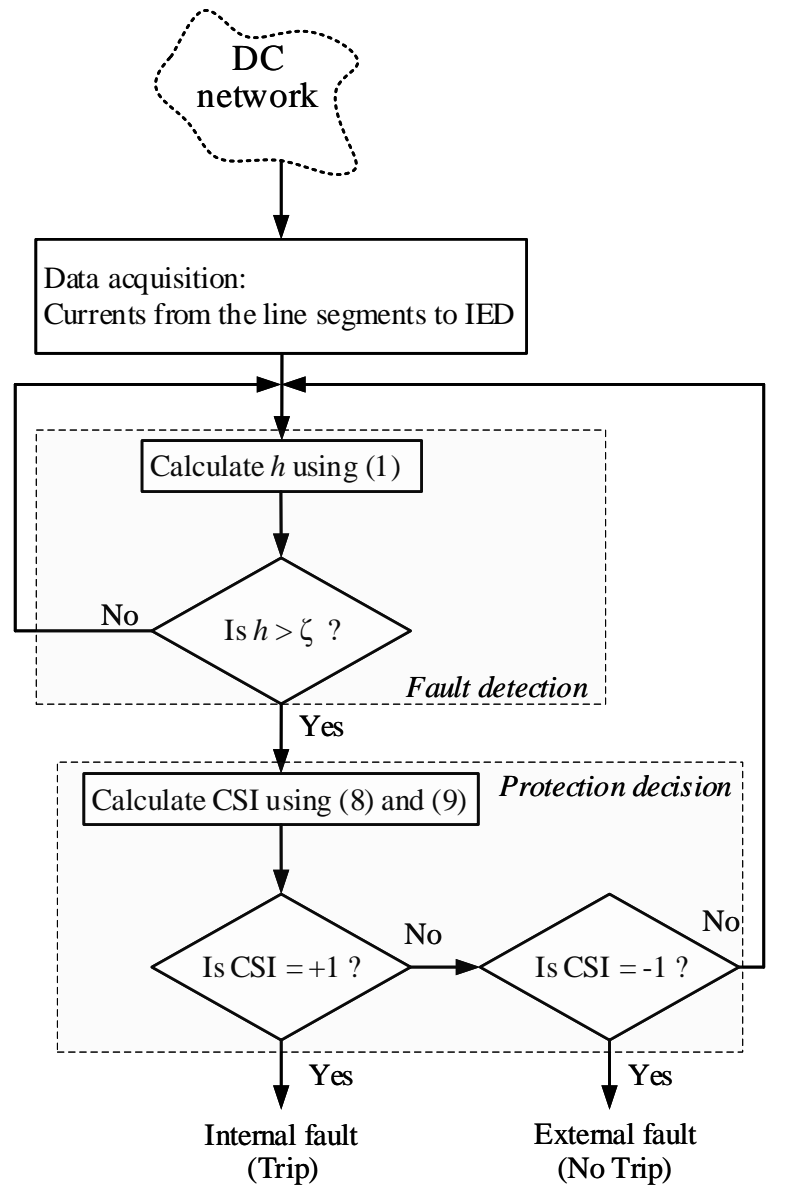

Fig. 11. Flow diagram of proposed protection algorithm.

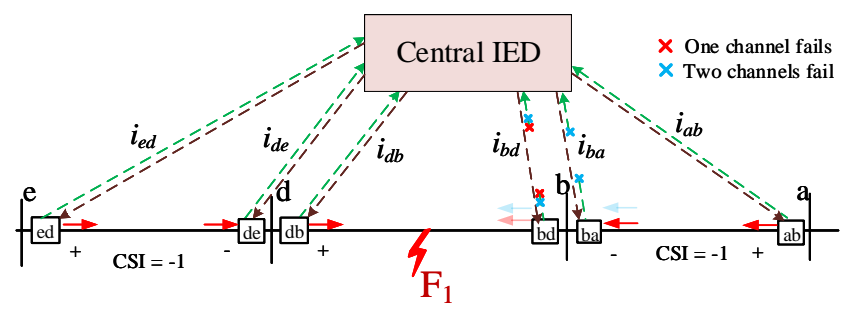

Fig. 12. Performance during communication links failure.

fails in the microgrid or two channels fail (one from each nonadjacent segment). Further, when two or three channels fail from adjacent segments, two segments are identified as possible faulted segments. The selectivity of proposed method during communication link failure is listed in Table III.

For a fault on bus-b with communication link failure from the current sensor-bd, the $C S I$ for line segment-ab and

TABLE III

Selectivity With Communication Link FAILURE

\begin{tabular}{|l|l|}
\hline $\begin{array}{l}\text { Number of communication } \\
\text { channel fails }\end{array}$ & Segment isolation \\
\hline No channel & Only the faulted segment \\
\hline One channel & Faulted and the adjacent one \\
\hline Two channels & \\
\hline Three channels &
\end{tabular}

segment-ed are -1 as the bus-b fault is external to these segments. In case of the sum is greater than maximum load current at the bus-b, then the IED sends trip signal to SSCBbd. The fault is not yet cleared as the fault current is fed from segment-ab (high $\frac{d i_{a b}}{d t}$ ). Consequently, the IED sends trip signal to SSCB-ba and now the bus fault is cleared.

\section{G. Performance during communication delay}

There are different causes of communication delay, out of which time-out error between communication devices [25] and non-synchronous current sampling are common [11]. The change in currents at both ends of the line segment during fault in the presence of communication delay can be expressed as:

$$
X=\frac{1}{7} \sum_{n=1}^{7} x_{n} \quad \text { and, } Y=\frac{1}{7} \sum_{n+\epsilon_{n}=1}^{7+\epsilon_{n}} y_{n}
$$

where $\epsilon_{n}$ denote the delayed sample number (in this case, one lost sample corresponds to a delay of $250 \mu \mathrm{s}$ ). The proposed $C S I$ is obtained using the average value of current change over a window of $2 \mathrm{~ms}$ rather than use of samples in current differential technique. Thus, for communication delay (typically one or two samples, i.e. $0.5 \mathrm{~ms}$ for $4 \mathrm{kHz}$ sampling), the window length takes care of it. It can be inferred that the proposed strategy is resilient to communication delay.

\section{H. Performance with noisy signal}

The proposed CSI based protection method is immune to noisy signals. The average over window reduces the noise level in the signal to en extend and then the ratio of data windows is not affected by the noise. With noise in the current signal, (8) becomes

$$
\begin{aligned}
C S I & =\frac{X_{n o i s e} \cdot Y_{n o i s e}}{\left|X_{n o i s e}\right|\left|Y_{n o i s e}\right|}=\frac{\frac{1}{7} \sum_{n=1}^{7} x_{n \_n o i s e} \cdot \frac{1}{7} \sum_{n=1}^{7} y_{n \_n o i s e}}{\left|\frac{1}{7} \sum_{n=1}^{7} x_{n \_n o i s e}\right|\left|\frac{1}{7} \sum_{n=1}^{7} y_{n \_n o i s e}\right|} \\
& =\frac{\sum_{n=1}^{7} x_{n \_n o i s e} \cdot \sum_{n=1}^{7} y_{n \_n o i s e}}{\left|\sum_{n=1}^{7} x_{n \_n o i s e}\right|\left|\sum_{n=1}^{7} y_{n \_n o i s e}\right|}
\end{aligned}
$$

From (11), it is clear that the combination of averaging and CSI computation for a corresponding window of $\frac{d i}{d t}$ results in accurate identification of fault even with noisy situation.

\section{Simulation Results}

The proposed algorithm is tested for the $350 \mathrm{~V} \mathrm{DC} \mathrm{mi-}$ crogrid shown in Fig. 1. The system components and their parameters are provided in Table IV. Using PSCAD/EMTDC simulation, faults are created in different line segments and data are acquired at a sampling rate of $4 \mathrm{kHz}$. The performance of the method is tested for several cases, e.g. fault at different locations, communication link failure, high resistance fault and communication delay. 
TABLE IV

DC MICROGRID COMPONENTS AND PARAMETERS[17]

\begin{tabular}{ll} 
System voltage & $350 \mathrm{~V} \mathrm{DC}$ \\
Base power & $30 \mathrm{~kW}$ \\
Battery & $96 \mathrm{~V}, 0.4 \mathrm{kAh}$ \\
Battery DC-DC converter & $10 \mathrm{~kW}$ \\
Solar panel & $V_{m p}=54.7 \mathrm{~V}, I_{m p}=5.58 \mathrm{~A}$ at STC \\
PV converter & $10 \mathrm{~kW}$ \\
VSC connecting to utility grid & $15 \mathrm{~kW}$ \\
Load & Constant impedance load, $30 \mathrm{~kW}$ \\
Fault resistance & $0-10 \Omega$ \\
DC link capacitor & $2.5 \mathrm{mF}$ \\
Cable parameters: & \\
Cross-section area & $240 \mathrm{~mm}$ \\
Resistance & $0.125 \Omega / \mathrm{km}$ \\
Inductance & $0.232 \mathrm{mH} / \mathrm{km}$ \\
Capacitance & $0.67 \mu \mathrm{F} / \mathrm{km}$ \\
\hline
\end{tabular}

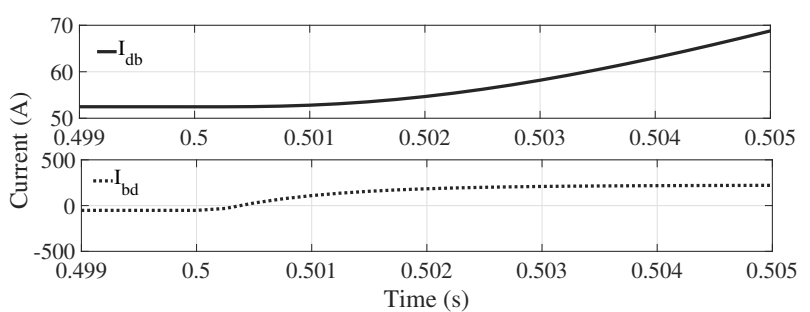

(a)

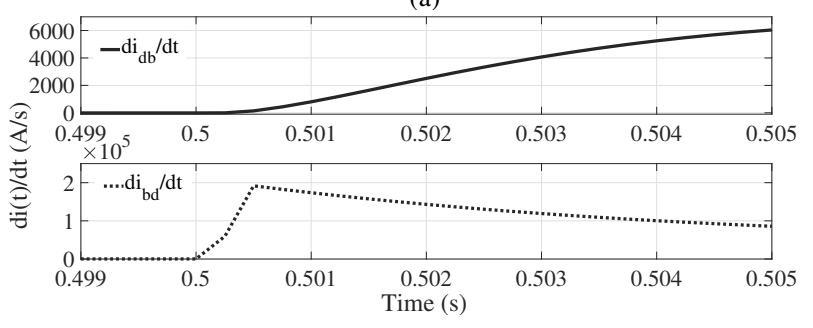

(b)

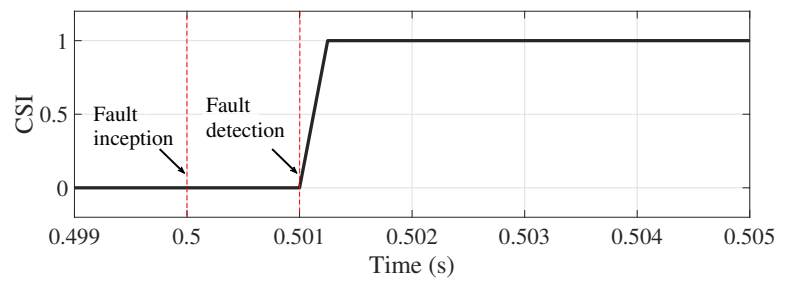

(c)

Fig. 13. For the internal fault $F_{1}$ in the line segment-db (a) current at $\mathrm{d}$ and b sides (b) rate of change in current (c) the corresponding $C S I$.

\section{A. Fault identification and protection decision}

A pole-to-ground fault $\left(F_{1}\right)$ with a fault resistance of $5 \Omega$ is simulated at $0.5 \mathrm{~s}$ in the middle of the line segment-bd (Fig. 1). The currents and their rate of change seen by the sensors at SSCB-db and SSCB-bd are shown in Fig. 13(a) and Fig. 13(b) respectively. As shown in Fig. 13(c), the average in the change of current in a window at both ends ( $\mathrm{X}$ and $\mathrm{Y}$ ) is found to be positive, which results in $C S I$ to be +1 using (8).

Similarly, an external fault $F_{2}$ (in the system in Fig. 1) with a fault resistance of $5 \Omega$ is created at $0.5 \mathrm{~s}$. The currents seen by sensors at SSCB-db and SSCB-bd are shown in Fig. 14(a). Further, it can be seen in Fig. 14(b) that the signs of change in current over a window at both ends of the line segment-db are moving in opposite directions. As a result, it is confirmed

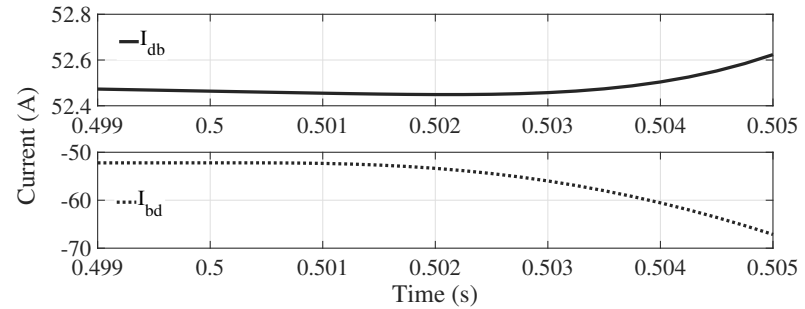

(a)

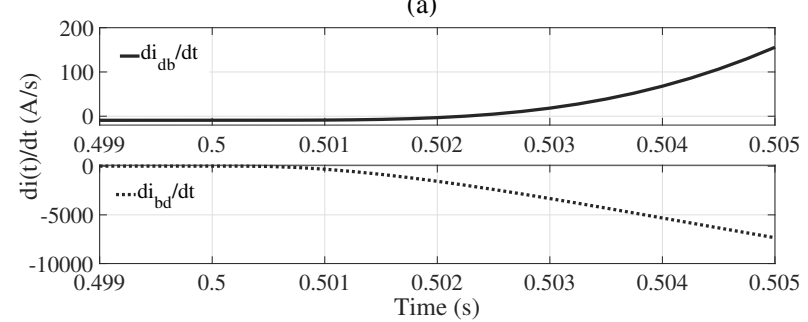

(b)

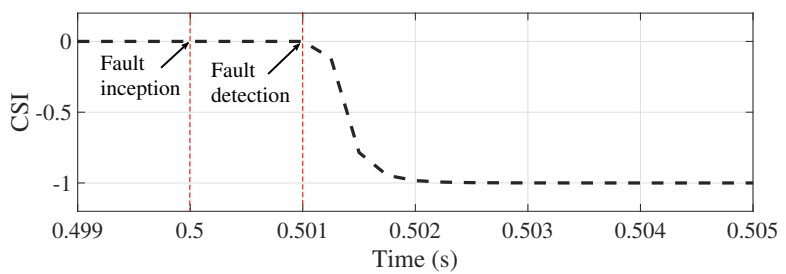

(c)

Fig. 14. For the external fault $F_{2}$ in the line segment-ab (a) current at d and b sides (b) rate of change in current (c) the corresponding CSI.

from Fig. 14(c) that $C S I$ is -1 .

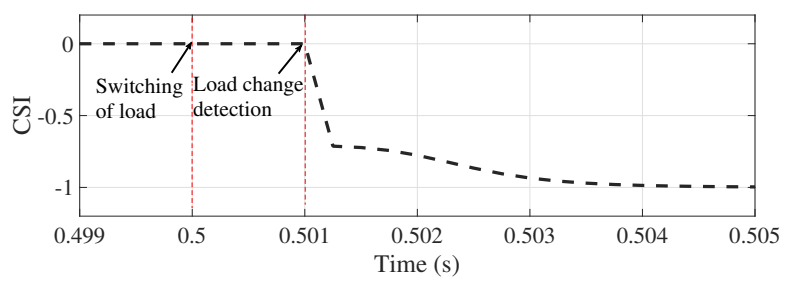

Fig. 15. CSI for sudden load change.

The performance of the method is also tested for sudden change in loading condition. The load is varied from $60 \%$ to $110 \%$ of rated value at bus-b. The $C S I$ is obtained as -1 as shown in Fig. 15. Therefore, no trip signal will be generated.

\section{B. Performance during communication link failure}

In case of one or two communication link failure, the proposed method is able to derive trip decision without losing proper selectivity. The influence of communication link failure is analyzed for various cases and the selectivity is provided in Table V.

\section{Comparative assessment}

1) Performance during high resistance internal fault: High resistance fault is a problem for available centralized protections based on current differential [11], [14], [15] and current direction based unit protection scheme [12] (refer section III). The proposed method uses the sign of the average of current 
TABLE V

Selectivity During Communication Failure for Fault $F_{1}$

\begin{tabular}{|l|l|l|l|}
\hline Events & Bus-d & Bus-b & Central IED \\
\hline $\begin{array}{l}\text { No communication } \\
\text { failure }\end{array}$ & $i_{d b}$ is available & $i_{b d}$ is available & X and Y are positive and $C S I=+1$ \\
\hline $\begin{array}{l}\text { One end communication } \\
\text { failure }\end{array}$ & $i_{d b}$ is not available & $i_{b d}$ is available & $\begin{array}{l}\text { Apply KCL at bus-d, satisfied } \\
\text { the criteria as in section IV-E }\end{array}$ \\
\hline $\begin{array}{l}\text { Both ends communication } \\
\text { failure }\end{array}$ & $i_{d b}$ is not available & $i_{b d}$ is not available & $\begin{array}{l}\text { Apply KCL at bus-d and b, satisfied } \\
\text { the criteria as in section IV-F }\end{array}$ \\
\hline $\begin{array}{l}\text { Both ends and one end from } \\
\text { adjacent lines fails }\end{array}$ & $i_{d b}$ is not available & $\begin{array}{l}i_{b d} \text { and } i_{b a} \text { are } \\
\text { not available }\end{array}$ & $\begin{array}{l}\text { Apply KCL at bus-d and a, satisfied } \\
\text { the criteria as in section IV-F }\end{array}$ \\
\hline Load change in bus-b & $i_{d b}$ is available & $i_{b d}$ is available & $\begin{array}{l}i_{d b}, i_{a b} \text { are positive and } \\
i_{b d}, i_{b a} \text { are negative, } C S I=-1\end{array}$ \\
\hline
\end{tabular}

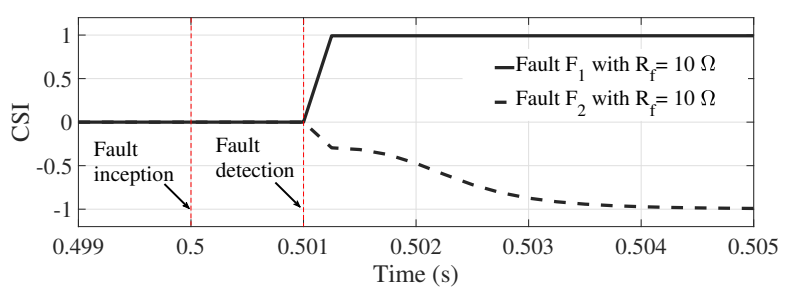

Fig. 16. Performance of proposed method during high resistance fault.

change in a window, which is independent of the current direction at an instant. The performance of the proposed method is evaluated for high resistance internal $\left(F_{1}\right)$ and external $\left(F_{2}\right)$ faults and the corresponding $C S I$ are found to be +1 and -1 respectively, as shown in Fig. 16.

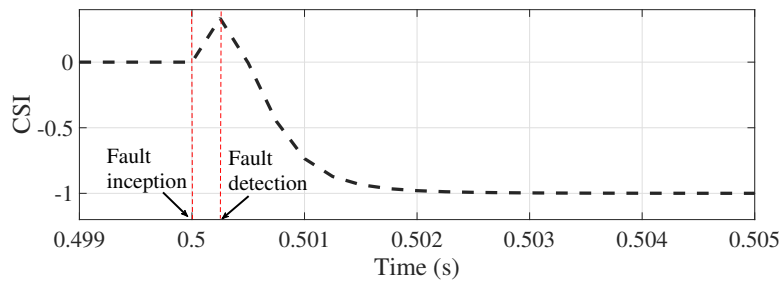

Fig. 17. CSI with communication delay during the fault in adjacent line a-b.

2) Performance in the presence of communication delay: At the middle of the line segment-ab, a PG fault $F_{2}$ is created with fault resistance of $2 \Omega$ at $0.5 \mathrm{~s}$ of simulation run. A communication delay of $1 \mathrm{~ms}$ is introduced in the current signal from the sensor at SSCB-bd of the line segment-db. Fort the fault $F_{2}$ (Fig. 1, the proposed method obtains $C S I$ using (8) as -1 (Fig. 17) which confirms an external fault and does not allow to trip the SSCBs.

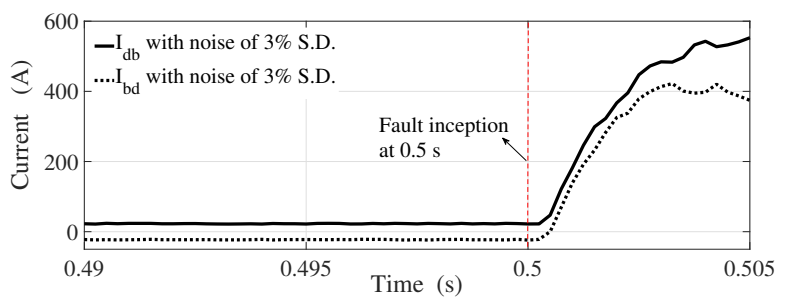

Fig. 18. Current signal with noise at both ends of the segment-db during $\mathrm{F}_{1}$ in Fig. 1.

\section{Performance of the proposed method with noisy signals}

The performance of the method is tested for the current signal contaminated with uniform distribution noise with zero mean and a standard deviation (S.D.) of $3 \%$ [17], [26]. During such a situation, currents $\mathrm{i}_{d b}$ and $\mathrm{i}_{b d}$ for a fault $\mathrm{F}_{1}$ in the line segment-db are shown in Fig. 18. With a fault resistance of 2 $\Omega$, a pole-to-ground fault is created at $0.5 \mathrm{~s}$. The corresponding $\frac{d i}{d t}$ with and without considering moving average window is shown in Fig. 19. The $C S I$ is obtained (Fig 20) using the average $\frac{d i}{d t}$ output from the windows at both ends of the segmentdb. It is clearly observed that $C S I$ is +1 during the fault which is correct. Therefore, the proposed method performs well even during noisy condition by using the averaging and $C S I$ techniques.

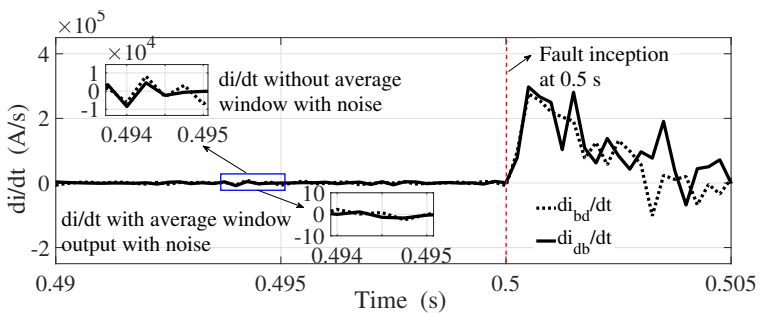

Fig. 19. $\frac{d i}{d t}$ of the currents in Fig. 18 with and without moving average window.

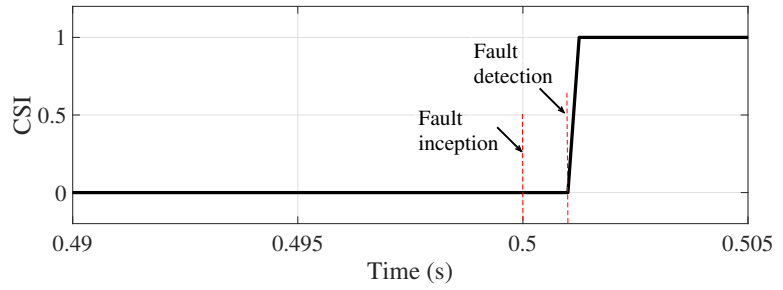

Fig. 20. CSI obtained from the signals with noise.

\section{EXPERIMENTAL RESULTS}

The proposed protection method has been experimentally validated in a DC microgrid operating at a voltage reference $V_{d c_{\text {ref }}}$ of $75 \mathrm{~V}$ with $N=2$ buck converters, as shown in Fig. 21(a). Both the converters are tied radially to a programmable load (voltage-dependent mode). Each converter is controlled by dSPACE MicroLabBox DS1202 (target), with control commands from the ControlDesk from the PC (host). 


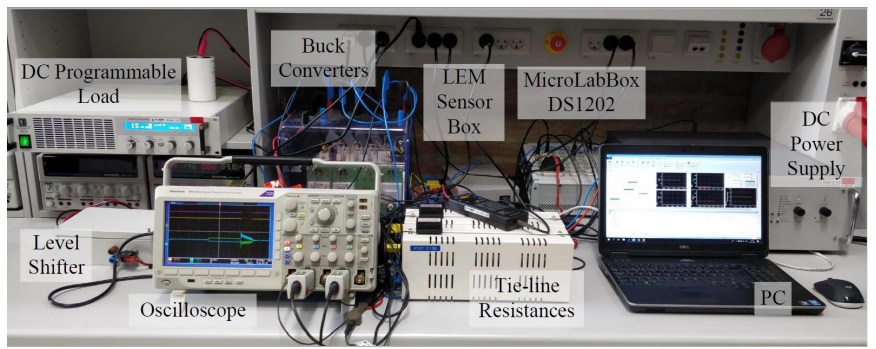

(a)

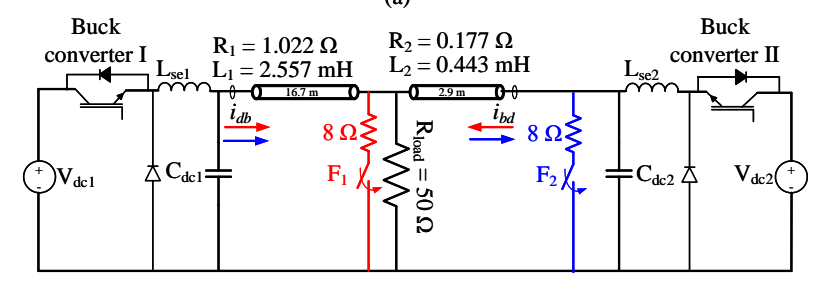

(b)

Fig. 21. (a) Experimental setup on a scaled-down DC microgrid (b) single line diagram of the setup.

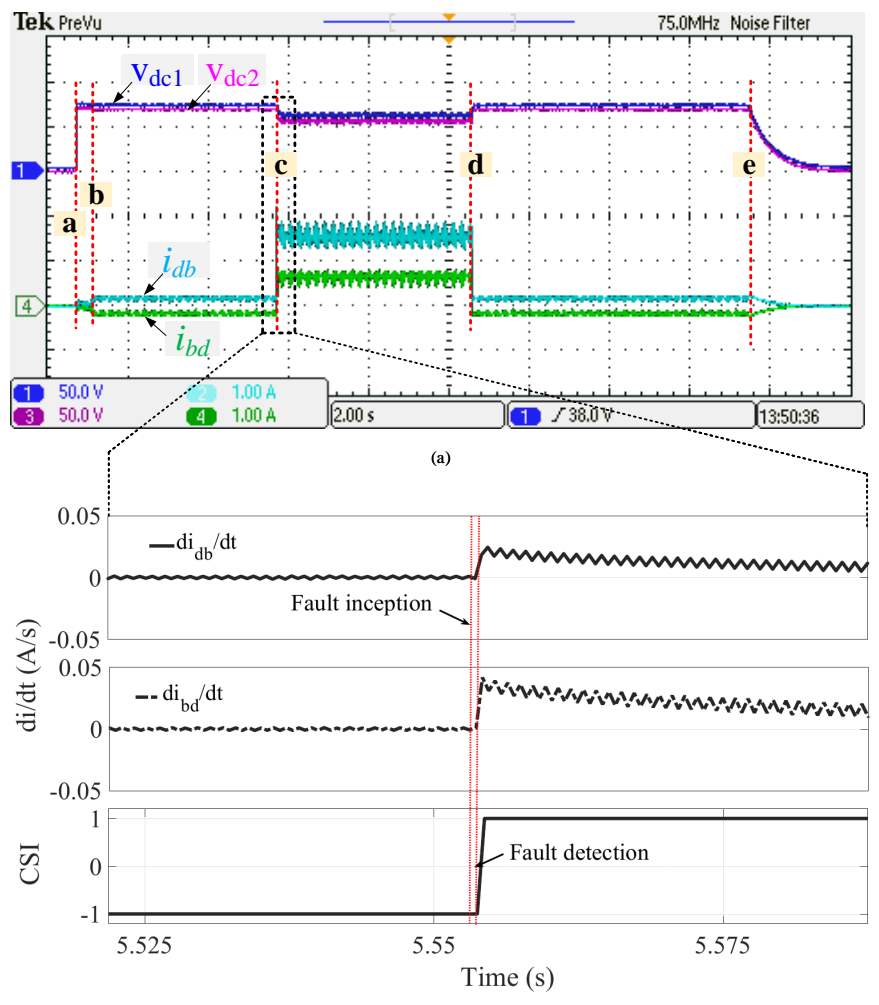

(b)

Fig. 22. (a) Performance of the proposed protection strategy in experimenta setup for internal and external faults, (b) Zoomed version of rate of change of current to calculate $C S I$ during transition from external to internal fault.

A simplified single line diagram of the experimental setup is shown in Fig. 21(b).

Using the tie-line current measurements $i_{d b}$ and $i_{b d}$, the proposed CSI based protection scheme is designed in the control platform to detect internal and external faults. The experimental testbed parameters are provided in Appendix.

As already established in simulated environment, it can be seen in Fig. 22(a) that the tie-line currents are opposite during the external fault $F_{2}$ (as indicated in Fig. 21(b)) during the event b after the switching pulses are initiated at event a. This validates the calculated $C S I$ using (9) to be -1 during external faults, which can be seen in the zoomed figure for the rate of change of ti-line currents in Fig. 22(a) before event c. Further during event c, an internal fault $F_{1}$ (as indicated in Fig. 21(b)) is subjected, where the currents rise in the same direction. Prior to this fault, it can be seen in Fig. 22(b) that $C S I$ jumps to 1 to confirm the presence of an internal fault as per the proposed strategy. Further when the internal fault is removed during event $\mathbf{d}$, it can be seen in Fig. 22(a) that the tie-line currents $i_{d b}$ and $i_{b d}$ return back to $C S I$ being -1 . Finally, the converters are turned off during event e. Hence, the robustness of the proposed strategy is validated experimentally for both internal and external faults.

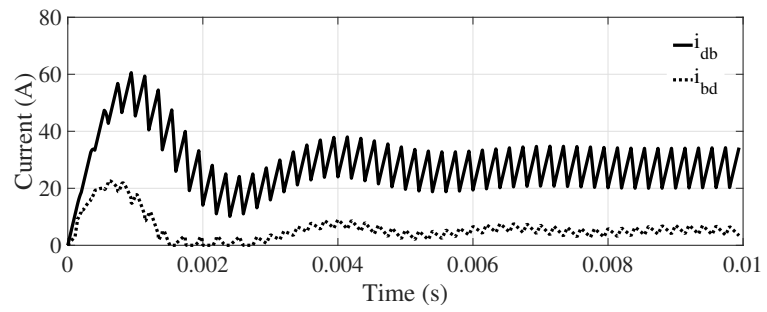

Fig. 23. High frequency ripples in currents from both converters in Fig. 21 switched at a frequency of $N_{s}=2 \mathrm{kHz}$.

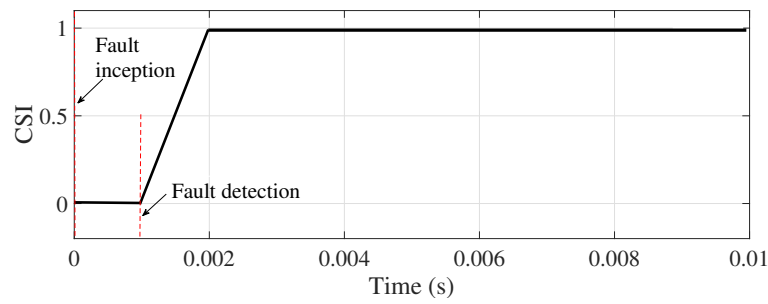

Fig. 24. Corresponding CSI for the study carried out in Fig. 23.

Further, the proposed method is also experimentally validated for currents with lower switching frequency of $2 \mathrm{kHz}$. A pole-to-ground fault is created during no load at $0 \mathrm{~s}$ and the currents from the DC-DC buck converters are captured as shown in Fig. 23. Under these conditions, it can be seen that the corresponding CSI moves to +1 as shown in Fig. 24, which establishes the robustness of the proposed strategy even for converters even with lower switching frequency.

\section{DISCUSSION}

In the proposed method, the fault detection does not take more than $1 \mathrm{~ms}$ as it uses 4 samples at a sampling frequency of $4 \mathrm{kHz}$ (using (1)). A window of $2 \mathrm{~ms}$ is used to calculate $C S I$, thus the protection decision can be taken in $3 \mathrm{~ms}$ of the fault inception which is evident from the results. The average of current change over a window is considered to avoid the issues associated with communication delay and any spurious data in the signal. As an advantage, the proposed method identifies the exact faulted segment during a communication channel fails. Furthermore, the faulted segment along with an adjacent one are isolated in case of two or three channels fail. The 
method can be applied to any DC microgrid including ring bus, grounded and ungrounded systems as it uses current signal of each line segment.

\section{CONCLUSION}

In this work, a centralized DC microgrid protection scheme is proposed. The similarity of rate of current change over a window of both ends of the line segment identifies an internal fault. IEC 61850 protocol is realized for the communication in DC microgrid application using SimEvents blocks. The performance of the protection scheme is tested for high resistance fault, communication delay and link failure. The protection decision is taken within $3 \mathrm{~ms}$ or even faster in case of no delayed signals. This protection algorithm aids in power electronic based systems by providing an auxiliary signal to block the switching pulses being supplied to converter. As compared to available protection schemes that use both voltage and current data, the proposed protection scheme entails an economic alternative, which employs only one central IED for entire microgrid and one current sensor for each end of a line segment. Its performance is also validated under experimental conditions for both internal and external faults, and found accurate.

\section{APPENDIX}

The considered system consists of two sources with the converters rated equally for $600 \mathrm{~W}$. It should be noted that the controller gains are consistent for each converter.

Plant: $L_{s e_{i}}=3 \mathrm{mH}, C_{d c_{i}}=100 \mu \mathrm{F}, N_{s}=7.5 \mathrm{kHz}$

Controller: $V_{d c_{r e f}}=75 \mathrm{~V}, K_{P}^{H_{1}}=1.92, K_{I}^{H_{1}}=15, K_{P}^{H_{2}}=$ $4.5, K_{I}^{H_{2}}=0.08, T_{s w}=5 \mathrm{kHz}$.

\section{ACKNOWLEDGMENT}

The authors would like to thank Prof. Tomislav Dragicevic at Technical University of Denmark for initial comments, which has helped enhance the presentation of this paper.

\section{REFERENCES}

[1] R. Cuzner, "Does DC distribution make sense?," IEEE Electr. Mag., vol. 4, pp. 2-3, June 2016.

[2] S. Sahoo and S. Mishra, "A distributed finite-time secondary average voltage regulation and current sharing controller for DC microgrids," IEEE Trans. Smart Grid, vol. 10, no. 1, pp. 282-292, 2019.

[3] V. Kumar, S. R. Mohanty, and S. Kumar, "Event trigger super twisting sliding mode control for DC micro grid with matched/unmatched disturbance observer," IEEE Trans. Smart Grid, vol. 11, no. 5, pp. $3837-$ 3849, 2020.

[4] D. Salomonsson, L. Soder, and A. Sannino, "Protection of low-voltage DC microgrids," IEEE Trans. Power Del., vol. 24, pp. 1045-1053, July 2009.

[5] S. Augustine, M. J. Reno, S. M. Brahma, and O. Lavrova, "Fault current control and protection in a standalone DC microgrid using adaptive droop and current derivative," IEEE Journal of Emerging and Selected Topics in Power Electronics, available in early access, 2020.

[6] A. Meghwani, S. Chakrabarti, S. C. Srivastava, and S. Anand, "Analysis of fault characteristics in DC microgrids for various converter topologies," in 2017 IEEE ISGT-Asia, pp. 1-6, Dec 2017.

[7] X. Feng, L. Qi, and J. Pan, "A novel fault location method and algorithm for DC distribution protection," IEEE Trans. Ind. Appl., vol. 53, pp. 1834-1840, May 2017.

[8] A. Meghwani, S. C. Srivastava, and S. Chakrabarti, "A non-unit protection scheme for DC microgrid based on local measurements," IEEE Trans. Power Del., vol. 32, pp. 172-181, Feb 2017.

[9] S. Azizi, M. Sanaye-Pasand, M. Abedini, and A. Hasani, "A travelingwave-based methodology for wide-area fault location in multiterminal DC systems," IEEE Trans. Power Del., vol. 29, pp. 2552-2560, Dec 2014.
[10] J. D. Park and J. Candelaria, "Fault detection and isolation in low-voltage DC-bus microgrid," IEEE Trans. Power Del., vol. 28, pp. 779-787, Jan. 2013.

[11] S. D. A. Fletcher, P. J. Norman, K. Fong, S. J. Galloway, and G. M. Burt, "High-speed differential protection for smart DC distribution systems," IEEE Trans. Smart Grid, vol. 5, pp. 2610-2617, Sept 2014.

[12] A. A. S. Emhemed, K. Fong, S. Fletcher, and G. M. Burt, "Validation of fast and selective protection scheme for an LVDC distribution network," IEEE Trans. Power Del., vol. 32, pp. 1432-1440, June 2017.

[13] A. N. Alndreev, E. A. Patrashkin, and A. S. Zheltov, "Centralized relay protection of power plants using iec-61850," in 2018 International MultiConference on Ind. Eng. and Modern Tech., pp. 1-3, Oct 2018.

[14] V. Nougain, S. Mishra, and A. K. Pradhan, "MVDC microgrid protection using a centralized communication with a localized backup scheme of adaptive parameters," IEEE Trans. Power Del., vol. 34, pp. 869-878, June 2019.

[15] M. Monadi, C. Gavriluta, A. Luna, J. I. Candela, and P. Rodriguez, "Centralized protection strategy for medium voltage DC microgrids," IEEE Trans. Power Del., vol. 32, pp. 430-440, Feb 2017.

[16] K. Jia, C. Wang, T. Bi, R. Zhu, and Z. Xuan, "Transient current waveform similarity based protection for flexible DC distribution system," IEEE Trans. Ind. Electr., vol. 66, pp. 9301-9311, Dec 2019.

[17] R. Mohanty and A. K. Pradhan, "Protection of smart DC microgrid with ring configuration using parameter estimation approach," IEEE Trans. Smart Grid, vol. 9, pp. 6328-6337, Nov 2018.

[18] S. Mohagheghi, J. Stoupis, and Z. Wang, "Communication protocols and networks for power systems-current status and future trends," in IEEE/PES PSCE, pp. 1-9, March 2009.

[19] T. Wang and A. Monti, "Fault detection and isolation in DC microgrids based on singularity detection in the second derivative of local current measurement," IEEE Journal of Emerging and Selected Topics in Power Electronics, available in early access, 2020.

[20] S. Ahmadi, I. Sadeghkhani, G. Shahgholian, B. Fani, and J. M. Guerrero, "Protection of LVDC microgrids in grid-connected and islanded modes using bifurcation theory," IEEE Journal of Emerging and Selected Topics in Power Electronics, available in early access, 2019.

[21] A. Ameli, K. A. Saleh, A. Kirakosyan, E. F. El-Saadany, and M. M. A. Salama, "An intrusion detection method for line current differential relays in medium-voltage DC microgrids," IEEE Trans. Inf. Foren. and Secur, vol. 15, pp. 3580-3594, 2020.

[22] R. Rana, S. Sahoo, S. Mishra, and J. C. H. Peng, "Performance validation of cooperative secondary controllers in autonomous AC microgrids under communication delays," in IEEE PESGM 2019, pp. 1-5, Aug 2019.

[23] A. Singhal, "Modern information retrieval: A brief overview," in IEEE Eng. Bull., pp. 35-43, vol. 24, no. 4, Jan. 2001.

[24] P. N. Tan, M. Steinbach, and V. Kumar, "Introduction to data mining," in IEEE Eng. Bull., pp. 500, ch. 8, MA, USA, 2001.

[25] N. Villamagna and P. A. Crossley, "A symmetrical component-based gps signal failure-detection algorithm for use in feeder current differential protection," IEEE Trans. Power Del., vol. 23, pp. 1821-1828, Oct 2008.

[26] J. G. Rao and A. K. Pradhan, "Power-swing detection using moving window averaging of current signals," IEEE Trans. Power Del., vol. 30 , pp. 368-376, Feb 2015.

Rabindra Mohanty (Member, IEEE) is currently a postdoctoral researcher in the division of Electric Power Engineering at Chalmers University of Technology, Gothenburg, Sweden. His research interests include power systems protection, renewable energy integration, protection and control of microgrids.

Subham Sahoo (Member, IEEE) is currently an Assistant Professor in the Department of Energy Technology, Aalborg University, Denmark. His research interests are control, optimization, and stability of microgrids, renewable energy integration, physics-informed AI tools for cyber-physical power electronic systems.

Ashok Kumar Pradhan (Senior Member, IEEE) is a Professor with the Department of Electrical Engineering, Indian Institute of Technology Kharagpur, India, since 2002. His research interests include Power System Relaying and Monitoring.

Frede Blaabjerg (Fellow, IEEE) is currently a Full professor in the Department of Energy Technology, Aalborg University, Denmark. His current research interests include power electronics and its applications, such as in wind turbines, PV systems, reliability, harmonics, and adjustable speed drives. 